\title{
Relationship between AC/DC Ratio and Light-blocking Structure for Reflective Photoplethysmographic Sensor
}

\author{
Hirofumi Nogami, ${ }^{1 *}$ Wataru Iwasaki, ${ }^{2}$ Nobutomo Morita, ${ }^{2}$ and Ryo Takigawa ${ }^{3}$ \\ ${ }^{1}$ Faculty of Engineering, Kyushu University, \\ 744 Moto-oka, Nishi-ku, Fukuoka City, Fukuoka 819-0395, Japan \\ ${ }^{2}$ Advanced Manufacturing Research Institute, National Institute of Advanced Industrial Science and Technology, \\ 807-1 Shuku-machi, Tosu, Saga 841-0052, Japan \\ ${ }^{3}$ Graduate School of Information Science and Electrical Engineering, Kyushu University, \\ 744 Moto-oka, Nishi-ku, Fukuoka City, Fukuoka 819-0395, Japan
}

(Received May 14, 2018; accepted December 12, 2018)

Keywords: wearable sensor, reflective photoplethysmographic sensor, health-monitoring sysytem

Photoplethysmographic (PPG) sensors are suitable for wearable devices, and they can provide a wide range of information such as stress level (calculated from the heart rate interval), respiration rate, heart rate, and blood vessel stiffness. Of particular importance is that reflective PPG sensors can be easily attached anywhere on the body with low wearer constraint. However, PPG sensors are susceptible to body motion artifacts. The output signal of PPG sensors is composed of alternating current (AC), originating from the heart cycle, and direct current (DC), originating from veins and stationary tissue. Motion artifacts affect DC signals, making it difficult to detect AC signals. Thus, it is important to reduce DC signals and increase the $\mathrm{AC} / \mathrm{DC}$ ratio. In this study, we investigated the effect of a light-blocking structure on the $\mathrm{AC} / \mathrm{DC}$ ratio. In addition, the $\mathrm{AC} / \mathrm{DC}$ ratio was estimated when the gap between the light source (LED) and the photodetector was small $(3.2 \mathrm{~mm})$ and large $(8.0 \mathrm{~mm})$. In this experiment, the measurement part was a fingertip, and the $\mathrm{AC} / \mathrm{DC}$ ratio was estimated when $\mathrm{AC}$ had the highest output with the force from step-by-step contact. As a result, the AC/DC ratio of the light-blocking structure was $2.4 \%$, and the $\mathrm{AC} / \mathrm{DC}$ of the non-light-blocking type was $0.9 \%$. Also, the $\mathrm{AC} / \mathrm{DC}$ of the small-gap PPG sensor was $2.4 \%$, and the $\mathrm{AC} / \mathrm{DC}$ of the large-gap sensor was $7.5 \%$. Thus, the light-blocking structure was effective in increasing the $\mathrm{AC} / \mathrm{DC}$ ratio, and a larger distance between the LED and photodetector was useful.

\section{Introduction}

For increased safety and security, wireless sensor network systems are being increasingly used in monitoring structural health, human health, agricultural field, and animal health. ${ }^{(1-6)}$ Structural health monitoring can improve the safety and reliability of buildings, bridges, tunnels, and express highways by detecting damage before it reaches a critical state. Damage is sensed by wireless sensor nodes installed on the structure. ${ }^{(1,2)}$ Human health monitoring detects

*Corresponding author: e-mail: nogami@mech.kyushu-u.ac.jp https://doi.org/10.18494/SAM.2018.1989 
sleep disorders, Parkinson's disease, and other ailments by logging a person's daily walking movements and posture using Global Positioning System (GPS) devices, triaxial accelerometers, and angular velocity sensors. ${ }^{(3)}$ These technologies have also been introduced in agricultural field monitoring, including animal health monitoring. ${ }^{(4-7)}$ It is believed that wireless sensor nodes attached to animals, in conjunction with wireless health-monitoring systems, can achieve early detection and prevention of diseases, and thus reduce economic loss.

In previous studies, wireless sensor nodes were attached to a chicken's wing to measure and transmit body temperature and activity data. ${ }^{(7)}$ The collected data was compared with previous avian-influenza epidemic data and used to monitor the health of an individual chicken. In order to construct this system, we developed several low-power technologies for the wireless sensor node, including a custom-built LSI for an event-driven system, a bimetal MEMS temperature switch, a miniaturized 300-MHz-band loop antenna, and polyvinylidene difluoride (PVDF) switches for activity sensors. ${ }^{(8-11)}$ The calculated average power consumption of the sensor nodes was less than $1 \mu \mathrm{W}$. Other groups have developed wearable wireless estrus detection sensors $^{(12)}$ and portable estrus intensity detection sensors. ${ }^{(13)}$ In this study, we focused on a photoplethysmographic (PPG) sensor.

Photoplethysmography has been widely and commonly used in a pulse monitor since it was developed by Hertzman in the 1930s. ${ }^{(14)}$ A particularly important feature of a reflective PPG sensor is that it can be easily attached with low wearer constraint, and it can detect a pulse wave from a body surface. It can provide a wide range of information, such as stress level calculated from the heart rate interval, respiration rate, heart rate, and blood vessel stiffness. ${ }^{(15)}$

The signal of a PPG sensor is composed of alternating current (AC) originating from the heart cycle and direct current (DC) originating from signals from veins and stationary tissue (see Fig. 1). ${ }^{(16)}$ The ratio of AC to DC signals is small, and this can be an issue when motion artifacts are superimposed on signals measured with a PPG sensor. When body movements occur, DC signals change because of changes in vascular volume or incident light. ${ }^{(16)}$ Furthermore, for measurements during exercise, $\mathrm{AC}$ can be unclear owing to body movement, because the acceleration of movement can overlap with the $\mathrm{AC}$ originating from the heat cycle. To calculate the pulse rate from the $\mathrm{AC}$ at the time of body movement, signal processing is performed on the basis of frequency or by using an acceleration sensor. However, there are many issues to be resolved in removing motion artifacts that are synchronized with the pulse rate.

Maeda's group have developed not only signal processing techniques but also approaches for improving hardware (sensor device). ${ }^{(17,18)}$ They reduced artificial noise of a PPG sensor attached to the upper arm by setting the attachment pressure to $30 \mathrm{mmHg}$ using a cuff during exercise. It is important to reduce the DC noise caused by body movement. ${ }^{(18)}$ Thus, we investigated the design structure of a reflective PPG sensor in which body motion artifacts would be suppressed.

In this study, we investigated a sensor light-blocking structure that increased the AC/DC ratio. We compared an unframed PPG sensor with a framed (light-blocking structure) sensor. In addition, the $\mathrm{AC} / \mathrm{DC}$ ratio was estimated when the gap between the light source [a lightemitting diode (LED)] and the photodetector was small $(3.2 \mathrm{~mm})$ and large $(8.0 \mathrm{~mm})$. In this experiment, the measurement part was a fingertip, and the AC/DC and AC/LED incident light ratios were estimated when $\mathrm{AC}$ had the highest output with the force from step-by-step contact. 


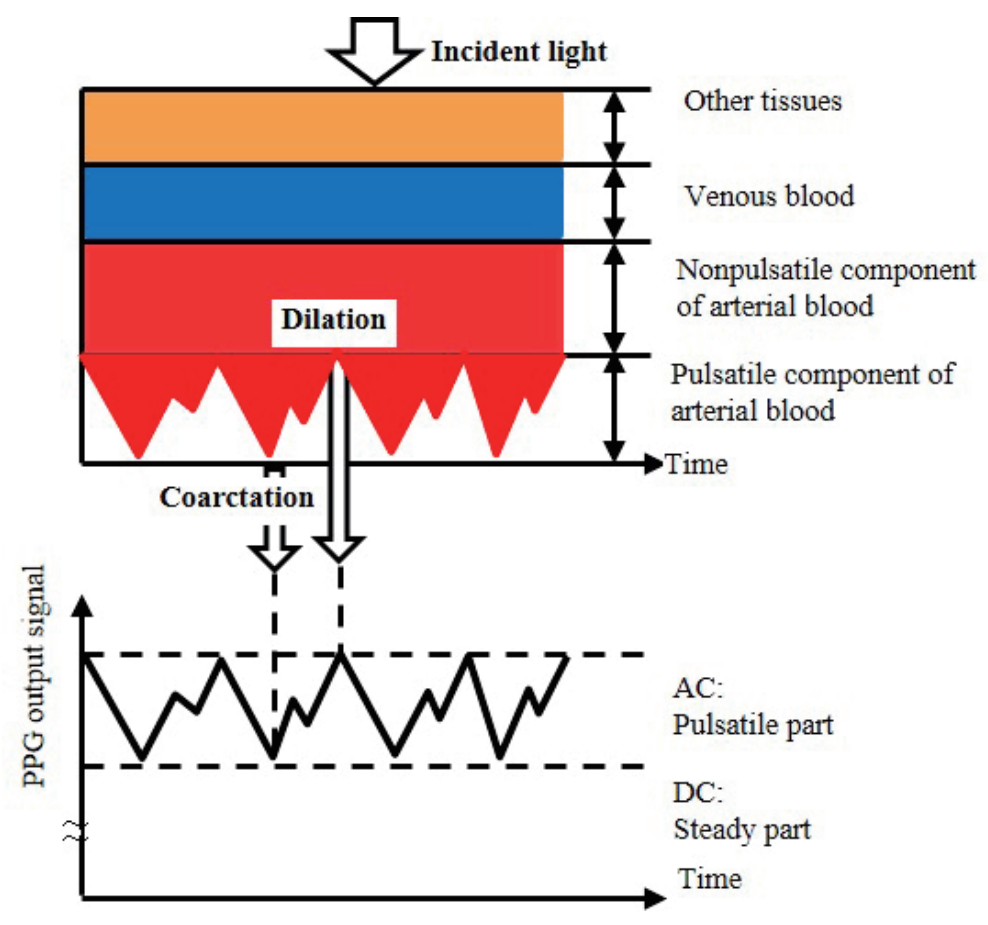

Fig. 1. (Color online) Measurement principle of PPG sensor.

\section{Device Structure and Its Working Principle}

This section concerns the device structure and working principle. First, the working principle of reflective PPG is indicated. Next, the device structure of a reflective PPG sensor is explained and then the fabricated PPG sensor is shown.

\subsection{Working principle of reflective PPG sensor}

Figure 2 shows the working principle of the reflective PPG sensor. The light from a LED is emitted into the skin tissue and scattered by it. The backscattered light from the body tissue is detected on a photodetector. When a blood vessel is dilated [see Fig. 2(a)], the incident light is absorbed partially by red blood cells, and the scattered light detected by the photodetector is small. When the blood vessel contracts [see Fig. 2(b)], the incident light is absorbed less by red blood cells, and the scattered light detected by the photodetector is large. Pulse waves can be detected by observing light intensity changes.

\subsection{Device structure and fabricated PPG sensor}

Figure 3 shows the device structure of the reflective PPG sensor. The device was composed of the light source, the photodetector, and the frame. The light source was an LED (peak wavelength, $940 \mathrm{~nm} ; 50 \%$ power angle, $120^{\circ}$ ), and the photodetector was a surface-mounted 
Photoplethysmographic sensor

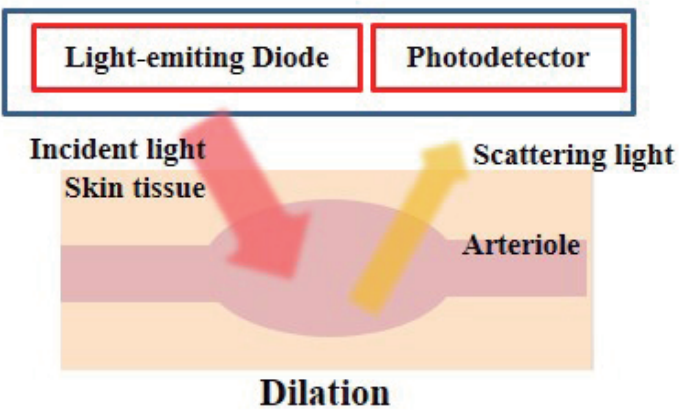

(a)
Photoplethysmographic sensor

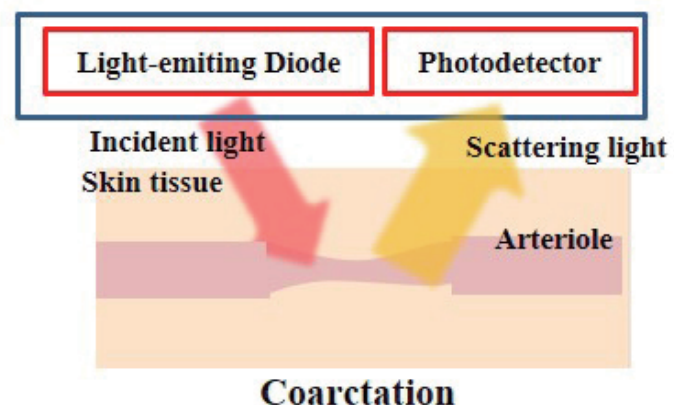

(b)

Fig. 2. (Color online) Working principle of the reflective PPG sensor. (a) Dilation and (b) coarctation.

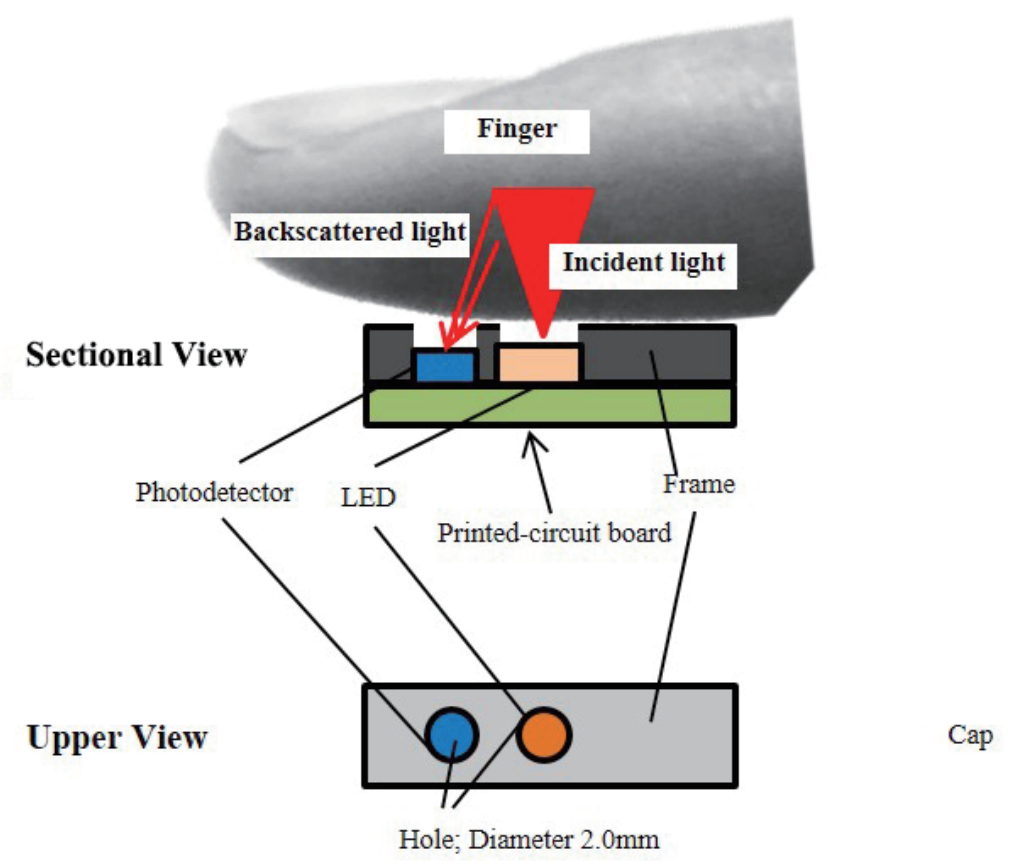

Fig. 3. (Color online) Device structure of a reflective PPG sensor.

phototransistor (peak wavelength, $880 \mathrm{~nm}$; directive property of half-value angle, $130^{\circ}$ ). The frame was made of aluminum, which prevented infrared light from passing through it, and it had 2.0-mm-diameter holes.

Three PPG sensors were used in the experiment, as shown in Fig. 4. The unframed one had a $3.2 \mathrm{~mm}$ gap between the LED and the photodetector without the frame [see Fig. 4(a)]. The small-gap one had a $3.2 \mathrm{~mm}$ gap between the LED and the photodetector with the frame [see Fig. 4(b)]. The large-gap one had a $8.0 \mathrm{~mm}$ gap between the LED and the photodetector with the frame [see Fig. 4(c)]. 


\section{Experiment}

Two kinds of experiments were conducted. In the first, we compared the $\mathrm{AC} / \mathrm{DC}$ ratio of the unframed PPG sensor [see Fig. 4(a)] with that of the small-gap PPG sensor [see Fig. 4(b)] to evaluate the light-blocking structure. Next, we compared the small- and large-gap PPG sensors to explore the effect of the distance between the light source and the photodetector.

As the Beer-Lambert Law is applied to human tissue with strong light scattering, the measurement depth increases as the distance between the light source and the detector increases. When measurement depth increases, a PPG sensor can prevent unwanted light from reaching the skin surface or the epidermis. However, as the amount of light received decreases, the distance between the LED and the photodetector increases. This causes increased power consumption by a PPG sensor, which conflicts with the low-power-consumption requirement for wearable sensors. Thus, we estimated the AC/DC and AC/LED ratios in the second set of experiments.

\subsection{Experimental task 1: Evaluating the light-blocking structure}

To evaluate the light-blocking structure, we compared the unframed PPG sensor [see Fig. 4(a)] with the small-gap (light-blocking structure) sensor [see Fig. 4(b)]. Figure 5 shows the experimental setup for evaluating AC/DC signals with measuring load. The PPG sensor was fixed on the force sensor (USL06-H5, Tec Gihan Co., Ltd.). The force sensor was set on the moving stage, and the moving stage was used to adjust it to the position where the finger was straight.

When the finger was placed on the PPG sensor, both the PPG sensor signal and the contact force between the finger and the PPG sensor could be recorded. We used the AC/DC ratio to measure the maximum AC signal, because $\mathrm{AC}$ is greatly affected by contact force (see Fig. 6). The measured AC/DC ratio is shown in Fig. 7.

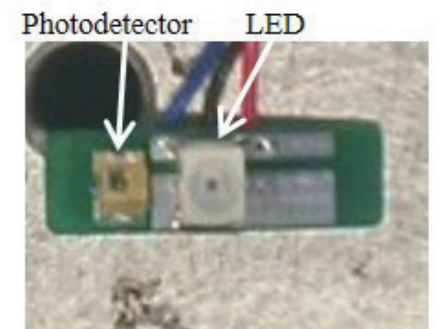

Unframed PPG sensor

(a)

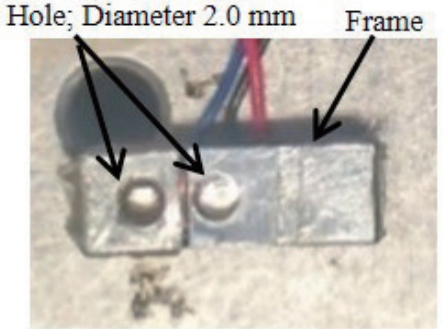

Small-gap PPG sensor

(b)

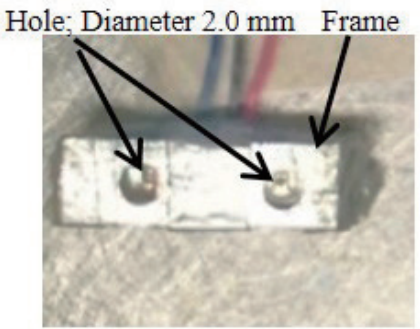

Large-gap PPG sensor

(c)

Fig. 4. (Color online) Photographs of the PPG sensors. (a) The unframed PPG sensor has a $3.2 \mathrm{~mm}$ gap between the LED and the photodetector without the frame. (b) The small-gap PPG sensor has a $3.2 \mathrm{~mm}$ gap between the LED and the photodetector with the frame. (c) The large-gap PPG sensor has an $8.0 \mathrm{~mm}$ gap with the frame. 


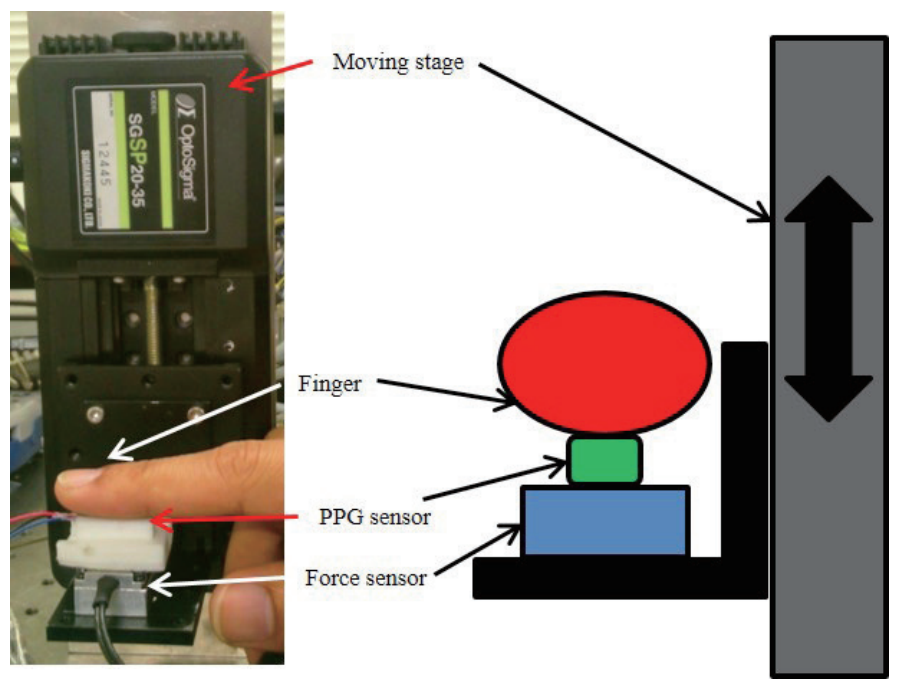

Fig. 5. (Color online) Experimental setup for evaluating AC/DC signals by measuring load.

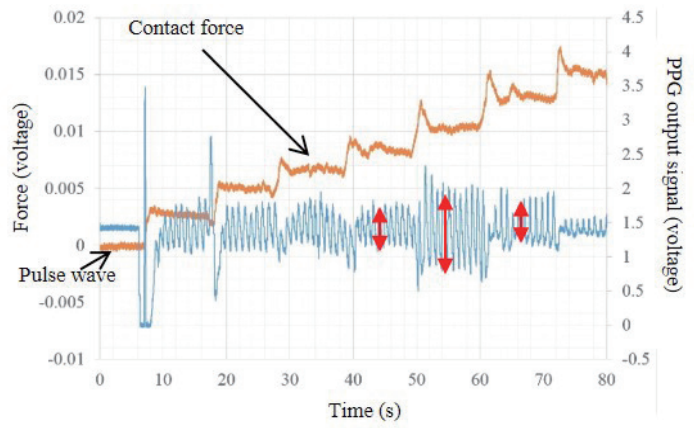

Fig. 6. (Color online) Relationship between AC signal and contact force.

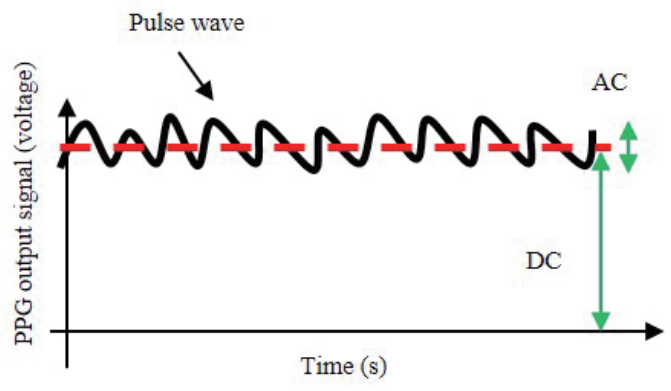

Fig. 7. (Color online) AC/DC ratio of PPG sensor.

\subsection{Experimental task 2: Evaluating the effect of the distance between the light source and the photodetector}

To evaluate the effect of the distance between the light source and the photodetector, we compared small-gap and large-gap PPG sensors (see Fig. 4). Using these sensors and the experimental setup (Fig. 5), both the pulse wave and contact force on the finger was measured.

The AC/DC ratio is shown in Fig. 7. We used the AC/DC ratio to measure the maximum $\mathrm{AC}$, as the AC signal was greatly affected by contact force (as shown in Fig. 6). Also, the AC/ LED power was estimated.

\section{Results and Discussion}

Figure 8 shows the AC/DC ratios of the three PPG sensors. The AC/DC ratio of the unframed PPG sensor was $0.9 \%$, that of the small-gap sensor was $2.4 \%$, and that of the largegap sensor was $7.5 \%$. 


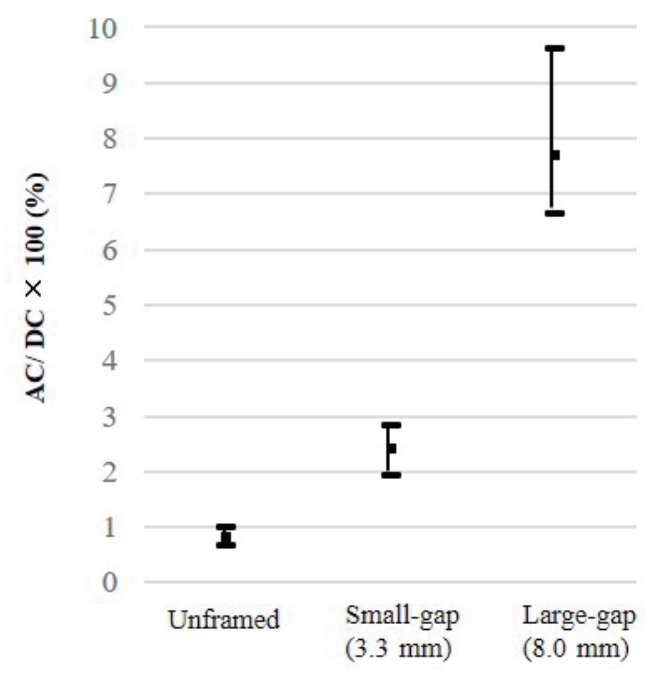

Fig. 8. AC/DC ratios of the unframed, small-gap, and large-gap sensors.

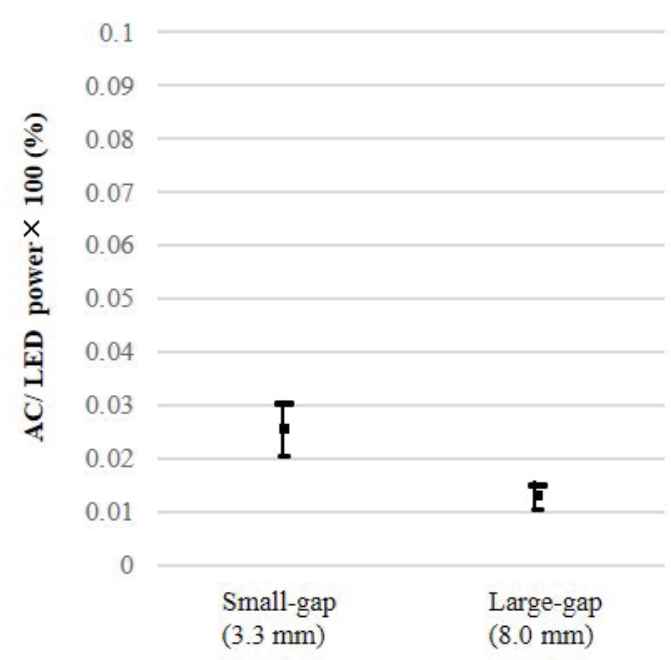

Fig. 9. AC/LED power ratios of both the small-gap and large-gap sensors.

To identify the effect of the light-blocking structure, we compared the unframed PPG sensor [see Fig. 2(a)] with the small-gap PPG senor [see Fig. 2(b)]. These sensors have the same distance between the light source and the photodetector. The frame (light-blocking structure) prevents unwanted light from reaching the skin surface or the epidermis, thereby increasing the AC/DC ratio. This result shows the effectiveness of the light-blocking structure.

To identify the effect of the distance between the LED and the photodetector, we compared the large- and small-gap PPG sensors [see Fig 2(c)]. As a result, we found that a larger distance between the light source and the photodetector increases the $\mathrm{AC} / \mathrm{DC}$ ratio. It is a concern that the light intensity from the artery relatively increases whereas that from the epidermis decreases, as the depth of measurement is a function of the distance between the light source and the photodetector. ${ }^{(19)}$ On the other hand, when comparing the light power, the AC/LED power for the small-gap sensor was $0.025 \%$ and that for the large-gap sensor was $0.013 \%$ (see Fig. 9). The large-gap sensor needs twice the LED power of the small-gap type.

\section{Conclusions}

We confirmed that reflective PPG sensors need a light-blocking structure to increase the AC/ DC ratio. Also, increasing the distance between the light source (LED) and the photodetector was effective in increasing the AC/DC ratio. However, this method increased LED power consumption, because the intensity of light received decreased. Thus, it is necessary to set the optimum distance considering both power consumption and the $\mathrm{AC} / \mathrm{DC}$ ratio.

\section{Acknowledgments}

This research was partially supported by grants from the Project of the Bio-oriented Technology Research Advancement Institution NARO (the research project for the future agricultural production utilizing artificial intelligence). 


\section{References}

1 B. J. Spencer, J. W. Park, K. A. Mechitov, H. Jo, and G. Agha: Procedia Eng. 171 (2017) 5.

2 T. Yamashita, H. Okada, T. Kobayashi, K. Togashi, D. Zymelka, S. Takamatsu, and T. Itoh: IEEE Sens. J. 16 (2016) 8840 .

3 A. Olivares, G. Olivares, F. Mula, J. M. Górriz, and J. Ramírez: J. Syst. Archit. 57 (2011) 905.

4 S. E. Díaz, J. C. Pérez, A. C. Mateos, M. Marinescu, and B. B. Guerra: Comput. Electron. Agric. 76 (2011) 252.

5 K. Nishihara, W. Iwasaki, M. Nakamura, E. Higurashi, T. Soh, T. Itoh, H. Okada, R. Maeda, and R. Sawada: IEEE Trans. Biomed. Eng. 60 (2013) 1645.

6 K. Suzuki, H. Okada, T. Itoh, T. Tada, M. Mase, K. Nakamura, and K. Tsukamoto: J. Virol. 83 (2009) 7475.

7 H. Okada, T. Itoh, K. Suzuki, and K. Tsukamoto: SENSORS, 2009 IEEE (IEEE, 2009) 1374.

8 H. Okada, T. Itoh, and T. Masuda: SENSORS, 2011 IEEE (IEEE, 2011) 1197.

9 Y. Zhang, A. Toda, H. Okada, T. Kobayashi, T. Itoh, and R. Maeda: 2012 IEEE 25th Int. Conf. Micro Electro Mechanical Systems (MEMS) (IEEE, 2012) 297.

10 H. Okada, T. Masuda, and T. Itoh: Procedia Eng. 25 (2011) 1028.

11 H. Nogami, H. Okada, S. Takamatsu, T. Kobayashi, R. Maeda, and T. Itoh: Jpn. J. Appl. Phys. 52 (2013) 9.

12 L.M. Andersson, H. Okada, R. Miura, Y. Zhang, K. Yoshioka, H. Aso, and T. Itoh: Comput. Electron. Agric. 127 (2016) 101.

13 W. Iwasaki, R. R. Sathuluri, O. Niwa, and M. Miyazaki: Anal. Sci. 31 (2015) 729.

14 A. B. Hertzman: Am. J. Physiol. 124 (1938) 328.

15 J. Allen: Physiol. Meas. 28 (2007) R1.

16 H. H. Asada, P. Shaltis, A. Reisner, S. Rhee, and R. C. Hutchinsonsada: IEEE Eng. Med. Biol. Mag. 22.3 (2003) 28.

17 Y. Maeda, M.Sekine, and T. Tamura: J. Med. Syst. 35 (2011) 829.

18 Y. Maeda, M. Sekine, and T. Tamura: J. Med. Syst. 35 (2011) 969.

19 S. Kashima, M. Nishihara, T. Kondo, and T. Ohsawa: Jpn. J. Appl. Phys. 31 (1992) 4097. 